University of New Hampshire

University of New Hampshire Scholars' Repository

Physics Scholarship

Physics

$4-1-2008$

\title{
Geotail and LFM comparisons of plasma sheet climatology: 1. Average values
}

T. B. Guild

Harlan E. Spence

Boston University, harlan.spence@unh.edu

\section{E. L. Kepko}

V. Merkin

J. G. Lyon

See next page for additional authors

Follow this and additional works at: https://scholars.unh.edu/physics_facpub

Part of the Physics Commons

\section{Recommended Citation}

Guild, T. B., H. E. Spence, E. L. Kepko, V. Merkin, J. G. Lyon, M. Wiltberger, and C. C. Goodrich(2008), Geotail and LFM comparisons of plasma sheet climatology: 1. Average values, J. Geophys. Res., 113, A04216, doi:10.1029/2007JA012611.

This Article is brought to you for free and open access by the Physics at University of New Hampshire Scholars' Repository. It has been accepted for inclusion in Physics Scholarship by an authorized administrator of University of New Hampshire Scholars' Repository. For more information, please contact Scholarly.Communication@unh.edu. 


\section{Authors}

T. B. Guild, Harlan E. Spence, E. L. Kepko, V. Merkin, J. G. Lyon, M. Wiltberger, and C. C. Goodrich 


\title{
Geotail and LFM comparisons of plasma sheet climatology:
}

\section{Average values}

\author{
Timothy B. Guild, ${ }^{1,2}$ Harlan E. Spence, ${ }^{1}$ E. Larry Kepko, ${ }^{1}$ Viacheslav Merkin, ${ }^{1}$ \\ John G. Lyon, ${ }^{1,3}$ Michael Wiltberger, ${ }^{4}$ and Charles C. Goodrich ${ }^{1}$ \\ Received 27 June 2007; revised 30 September 2007; accepted 5 February 2008; published 18 April 2008.
}

[1] We compare the statistics of central plasma sheet properties from 6 years of Geotail observations with 2 months of Lyon-Fedder-Mobarry (LFM) global MHD simulations. This statistical validation effort represents an inherently new method of systematically characterizing and quantifying global MHD model performance. For our comparison, we identify the central plasma sheet in the observations and simulation by identical criteria and select the simulation interval to ensure statistically similar distributions of solar wind conditions in both studies. After verifying our plasma sheet selection by inspecting the magnetic signatures of both studies, we compare the resultant number densities, thermal pressures, thermal energies, and bulk flows as functions of position across the equatorial plane. We find that the LFM model successfully reproduces the gross features of the global plasma sheet in a statistical sense. However, our comparison also reveals certain systematic discrepancies between the model and the observations. The LFM predicts a plasma sheet which is too dense, too cool, and exhibits faster globally averaged bulk flows than the observed plasma sheet. By quantifying the LFM overestimate of ionospheric transpolar potential and showing that $\Phi_{P C}$ correlates with plasma sheet flow speed, we demonstrate that $15 \%$ of the plasma sheet velocity discrepancy is reflected in a $\Phi_{P C}$ overestimate. This statistical validation effort represents an essential first step toward the rigorous, quantitative evaluation of a global MHD model in the plasma sheet.

Citation: Guild, T. B., H. E. Spence, E. L. Kepko, V. Merkin, J. G. Lyon, M. Wiltberger, and C. C. Goodrich (2008), Geotail and LFM comparisons of plasma sheet climatology: 1. Average values, J. Geophys. Res., 113, A04216, doi:10.1029/2007JA012611.

\section{Introduction}

[2] The Earth's magnetotail plasma sheet is an important, dynamic link in the coupled solar-terrestrial system. Processes in the plasma sheet modulate the transfer of solar wind mass and energy into the inner magnetospheric plasma population, thereby mediating solar wind geoeffectiveness. Despite its importance, the dynamic and structured nature of the plasma sheet has hindered our ability to understand and model its global properties.

[3] Previous studies relied on comprehensive spacecraft data sets to accumulate a time-averaged characterization of the global plasma sheet. These studies constructed plasma sheet databases along the midnight meridian [e.g., Spence et al., 1989], in or mapped to the equatorial plane [e.g., Angelopoulos et al., 1993; Wing and Newell, 1998], or parameterized as a function of incident solar wind param-

\footnotetext{
${ }^{1}$ Center for Space Physics, Boston University, Boston, Massachusetts, USA.

${ }^{2}$ Now at The Aerospace Corporation, Chantilly, Virginia, USA.

${ }^{3}$ Also at Department of Physics and Astronomy, Dartmouth College, Hanover, New Hampshire, USA.

${ }^{4}$ High Altitude Observatory, National Center for Atmospheric Research, Boulder, Colorado, USA.
}

Copyright 2008 by the American Geophysical Union. 0148-0227/08/2007JA012611 eters [Tsyganenko and Mukai, 2003]. These plasma sheet models, representing state-of-the-art plasma sheet characterizations of their time, are empirical, not physics-based. Despite their utility, they provide only spatial and timeaveraged glimpses of an inherently structured and dynamic region and without internal physical self-consistency. Furthermore, despite the large volumes of data, the plasma sheet remains an undersampled region, thus limiting the range of validity and reliability of any empirical model.

[4] The ability to predict time-dependent, spatially evolving plasma sheet characteristics may significantly improve our forecasting of certain space weather effects near the Earth. For example, the plasma sheet is often considered a major seed population for energetic particles trapped in the inner magnetosphere, which in turn pose a direct threat to astronauts or technological systems operating in the nearEarth space environment. Although data-driven local-linear filters [e.g., Vassiliadis et al., 1995] and neural networks [e.g., Gleisner and Lundstedt, 1997] have been shown to effectively predict space weather effects using empirical methods, global magnetohydrodynamic (MHD) models of the magnetosphere provide a physics-based, time-dependent specification of plasma sheet characteristics throughout the entire simulation volume. These simulations possess predictive promise through physics-based causal relationships, a feature lacking in empirical models. 
[5] However, before a global MHD model can provide a useful prediction, it must be validated with existing data. In this paper, we extend a previous effort [Guild et al., 2004] by validating a 2-month global MHD simulation of the equatorial plasma sheet with 6 years of Geotail observations. We first demonstrate that both the observations and simulations include similar magnetospheric states by comparing the distributions of solar wind driving parameters. We compare and contrast the resultant "climatology" of the Lyon-Fedder-Mobarry (LFM) MHD model with global average maps of Geotail plasma sheet measurements. We define plasma sheet "climatology" as those global, underlying characteristics and trends which persist after months to years of time averaging. This climatology can be related to the short-duration, time-dependent "space weather" within the plasma sheet in direct analogy to the relationship between terrestrial climate and weather. We explore and quantify some of the more apparent climatological discrepancies, and identify possible sources of systematic differences. This effort begins the iterative validation process of model assessment and improvement which will ultimately lead to the improved predictive ability of the LFM global MHD model.

[6] In this paper we compare equatorially averaged maps of plasma and field parameters from Geotail observations and LFM simulations and assess the model performance in the central plasma sheet during typical solar wind intervals. We concentrate on the thermal pressure and convective velocity distributions due to their association with the global structure of the tail and plasma sheet transport, two important tests of the LFM magnetosphere. We first describe the plasma sheet and solar wind observations used in section 2 and the LFM simulations performed in section 3 . We justify the statistical comparison of 6 years of Geotail observations with 2 months of LFM simulation through the use of a statistical similarity argument in section 4 . We then present comparisons of equatorial maps of the magnetic field, plasma parameters, and bulk flows in section 5. After finding a systematic discrepancy in the bulk flow speeds of the observations and simulation, we identify and quantify the potential sources of the discrepancy in section 6 and summarize with the most important findings of our comparison in section 7 .

\section{Plasma Sheet and Solar Wind Observations}

[7] We used data from the Low Energy Particle (LEP) [Mukai et al., 1994] and Magnetic Field (MGF) [Kokubun et al., 1994] experiments on the Geotail satellite. The DARTS/GEOTAIL online database at the Institute of Space and Astronautical Science (ISAS) provided 12-s cadence LEP and 3-s cadence MGF data from January 1995 through December 2000. We linearly interpolated all data to a common 12-s time resolution and calculated derived parameters such as the thermal pressure $\left(P_{t h}=n k T\right)$, the magnetic pressure $\left(P_{m a g}=\frac{B^{2}}{2 \mu_{0}}\right)$ and their ratio, the plasma beta $\left(\beta=\frac{P_{t h}}{P_{\text {mag }}}\right)$. In these formulas, $n$ is the ion number density, $T$ is the ion temperature, and $B$ is the magnetic field magnitude. We assumed that the electron contribution to thermal pressure is small $(\leq 15 \%)$ [Spence et al., 1989] and consider only the total ion pressure.
[8] From these observations, we constructed climatological maps of the macroscopic plasma properties of the plasma sheet. We included only Geotail measurements in the central plasma sheet, as determined by five criteria taken to be representative of this innermost plasma sheet region. The central plasma sheet is the volume closest to the neutral sheet, within which high-speed bulk flows transport magnetic flux, an important component of magnetotail dynamics. We first included only those observations taken antisunward of $X_{G S M}=-10 R_{E}$. Second, we included observations with ion thermal energies $(\mathrm{kT})$ greater than $1 \mathrm{keV}$. Here, we define $k T$ as the average of two diagonal components of the thermal energy tensor measured in spacecraft coordinates (nearly GSE) and provided by the DARTS Web site $\left(k T=k\left(\frac{T_{y y}+T_{z z}}{2}\right)\right)$. Third, we limited our data set to include only plasmas with $\beta>0.5$. Fourth, we required that the elevation angle of the magnetic field be sufficiently large $\left(\frac{\left|B_{z}\right|}{\sqrt{B_{X}^{2}+B_{Y}^{2}}}>0.5\right)$, a condition for identifying the central plasma sheet taken from Baumjohann et al. [1990]. This cutoff included all field elevation angles $>26^{\circ}$ from the equatorial plane. Finally, we required that observations be within $3 R_{E}$ of the location of a model neutral sheet. In our case, we used the Hammond et al. [1994] model. This is an empirical model parameterized by the solar wind dynamic pressure, which we computed from solar wind values ballistically propagated to the magnetosphere at the time of plasma sheet observations. We used WIND (SWE, MFI) plasma and field data before March 1998, and ACE (SWEPAM, MAG) thereafter, as our solar wind data. After eliminating anomalous observations (when the solar wind monitor is $<15 R_{E}$ upstream of the Earth, when $\left|V_{Y}\right|>$ $30 \%\left|V_{X}\right|$, or when $\left|V_{Z}\right|>20 \%\left|V_{X}\right|$ ), we ballistically propagated individual solar wind observations to the magnetosphere, rebinned to a uniform time series and smoothed with a 20 -min running average. We used these propagated and timesmoothed solar wind data points to compute the location of the neutral sheet at the times of the Geotail central plasma sheet observations.

[9] Table 1 summarizes the plasma sheet selection criteria. These limits have been taken, in part, from many previous studies of the plasma sheet [Baumjohann et al., 1990; Angelopoulos et al., 1994; Nishida et al., 1995; Nagai et al., 1998]. The inequality values strike a balance between selecting only those points closest to the neutral sheet yet including enough observations to yield meaningful statistics throughout the volume of interest. Approximately 530,000 data points at a 12-s cadence passed the selection criteria, cumulatively representing $\sim 77 \mathrm{~d}$ of plasma sheet measurements.

[10] We plot the spatial distribution of these points in Figure 1, with the equatorial $(X Y)$, meridional $\left(X Z_{N S}\right)$, and cross-tail $\left(Y Z_{N S}\right)$ planes in Figures $1 \mathrm{a}, 1 \mathrm{~b}$, and $1 \mathrm{c}$, respectively. For all plots we used the Geocentric Solar Wind (GSW) coordinate system, which is essentially the GSM coordinate system with the $\mathrm{X}$ axis aberrated to the direction of the solar wind velocity for each plasma sheet measurement. Tsyganenko and Fairfield [2004] provide a detailed discussion of the GSW coordinate system. We used the solar wind data previously propagated to the Earth, smoothed, and merged with our plasma sheet data set to determine solar wind time correspondence and the rotation matrix from GSM 
Table 1. Central Plasma Sheet Criteria for the Geotail and LFM Studies

\begin{tabular}{lc}
\hline \multicolumn{1}{c}{ Variable } & Value \\
\hline$T$ & $>1 \mathrm{keV}$ \\
$\beta$ & $>0.5$ \\
$X_{G S M}$ & $<-10 R_{E}$ \\
$\left|B_{Z}\right|$ & $>0.5^{\mathrm{a}}$ \\
$B_{X Y}$ & $<3 R_{E}{ }^{\mathrm{b}}$ \\
\hline$Z_{N S}=Z_{A C T U A L}-Z_{N S, M O D E L}$ &
\end{tabular}

${ }^{\mathrm{a}}$ After Baumjohann et al. [1990].

${ }^{\mathrm{b}} Z_{N S, M O D E L}$ is calculated using the Hammond et al. [1994] model neutral sheet.

to GSW. The magnetotail geometry tailward of the hinging distance is well ordered by the time-dependent GSW coordinate system, but individual orbital passes are obscured, as seen in Figure 1a. Note the complete and fairly uniform spatial coverage of the plasma sheet from $X_{G S W}=-10 R_{E}$ to the Geotail apogee, near $30 R_{E}$ in Figure 1a.

[11] The remaining panels of Figure 1 (Figures $1 \mathrm{~b}$ and 1c) illustrate the distance of all measurements from a model neutral sheet, with the ordinate axes defined as $Z_{N S}=$ $Z_{A C T U A L}-Z_{N S, M O D E L}$. We excluded measurements taken outside the $\pm 3 R_{E}$ limits of our central plasma sheet selection criteria (red lines). We also show the mean $Z_{N S}$ positions as functions of $X_{G S M}$ (Figure 1b) and $Y_{G S M}$ (Figure 1c), as green lines with error bars corresponding to the $1 \sigma$ standard deviation from the mean. Our data set shows almost no sampling bias with increasing distance down the tail (the mean $Z_{N S}$ green line is essentially flat with decreasing $X_{G S M}$, Figure $1 \mathrm{~b}$ ) but shows a noticeable bias toward higher (positive) $Z_{N S}$ values at the dusk flank (for large $Y_{G S M}$, Figure 1c). Despite this bias, the mean value of $Z_{N S}$ is only $0.2 R_{E}$, with a standard deviation of $1.5 R_{E}$ for all $X_{G S M}, Y_{G S M}$ positions.

[12] The plasma sheet data set was designed for its global, statistical coverage. The data set comprises $\sim 530,000$ data points, but more importantly, when binned into $3 \times 3 R_{E}$ bins, yields an average of $\sim 15$ independent, separate Geotail passes per bin. The data set has shortcomings that warrant caution. Since the global coverage comes from Geotail's changing orbital orientation relative to the GSW coordinate system throughout the year, plasma sheet sampling is a function of season. Geotail predominantly measured the duskside of the plasma sheet between September and December and the dawnside between January and March. Geotail apogee in December determined the antisunward extent of our data set and provided the highest spatial density of plasma sheet measurements. Regardless, we are confident that our Geotail data set samples the plasma sheet uniformly because it yields a reasonable plasma sheet magnetic configuration, as will be shown in section 5.1. Additionally, our data set reproduces equatorial plasma distributions found in the literature, which were generated with different satellites as well as different sorting criteria, as will be shown in section 5.2.

\section{Lyon-Fedder-Mobarry Global MHD Simulations}

[13] We compared our Geotail data set described above with a comparable plasma sheet simulation obtained from the Lyon-Fedder-Mobarry global MHD model. The LFM
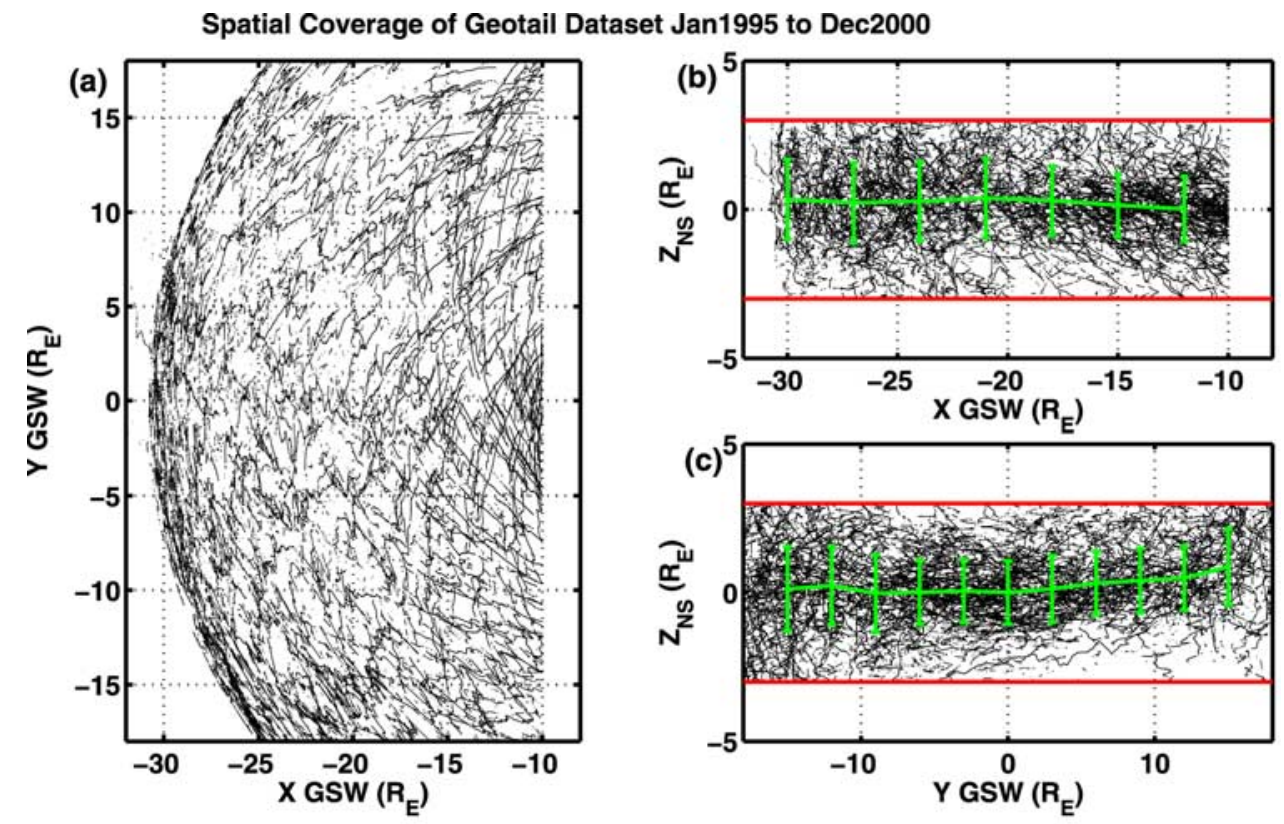

Figure 1. Scatterplot of the spatial coverage of all central plasma sheet samples of our Geotail data set. Shown are the (a) $X Y$, (b) $X Z_{N S}$, and (c) $Y Z_{N S}$ planes, where $Z_{N S}=Z_{\text {actual }}-Z_{\text {model }}$. The $X$ and $Y$ dimensions are given in $G S W$ coordinates, and the red lines indicate the $\pm 3 R_{E}$ limits which exclude measurements far from the Hammond et al. [1994] neutral sheet. The green lines in Figures 1b and 1c indicate the average $Z_{N S}$ position as a function of the abscissa. Error bars correspond to $1 \sigma$ about the mean. 


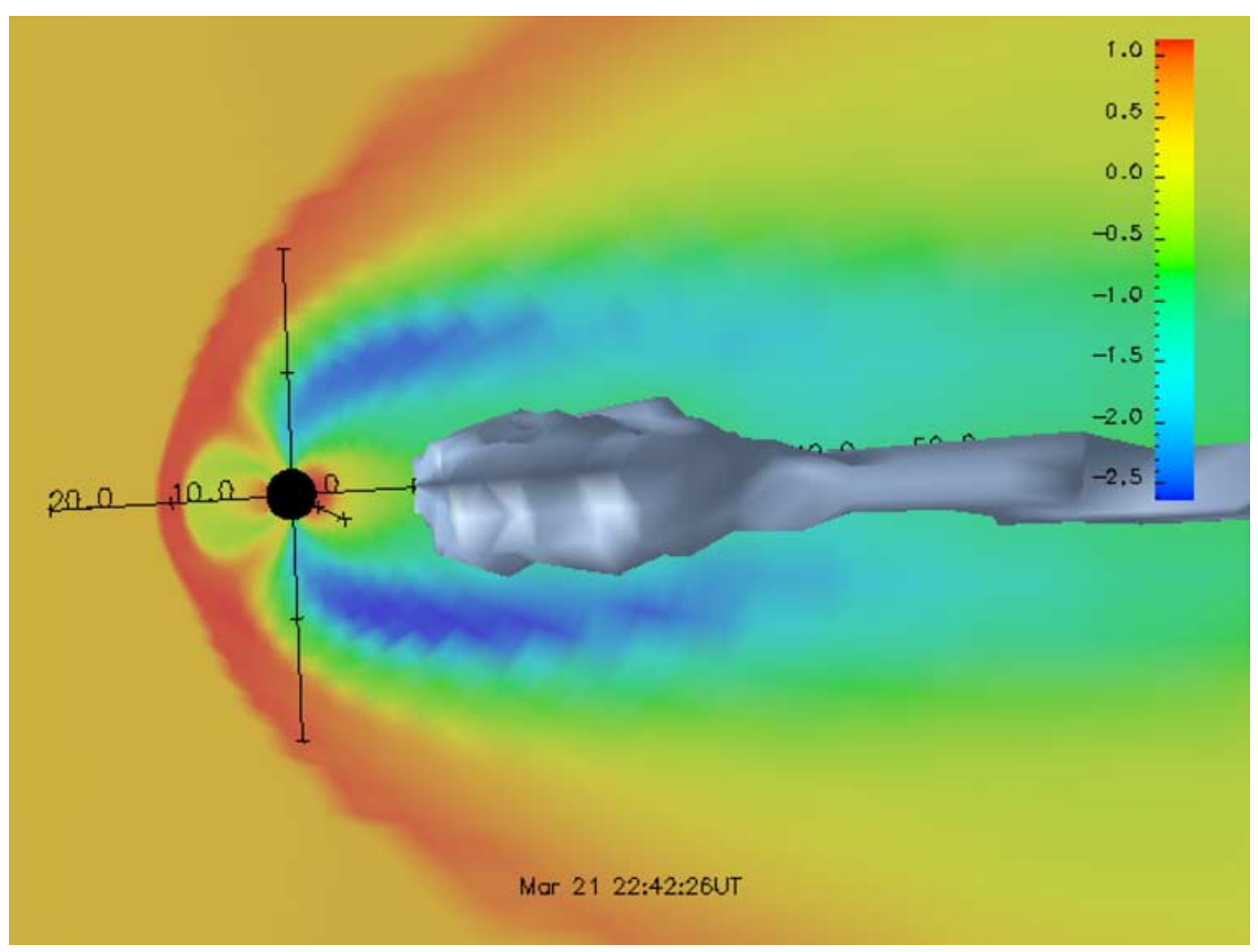

Figure 2. A representative time step of the LFM simulation showing the central plasma sheet boundary as the gray surface in the tail. The view is roughly from dusk and the noon-midnight plane is colored in $\log$ (Density) from $0.001 \mathrm{~cm}^{-3}$ in blue to $10 \mathrm{~cm}^{-3}$ in red. Black GSM coordinate axes have tick marks every $10 R_{E}$.

solves the three-dimensional ideal MHD equations selfconsistently on a stretched spherical grid centered on the Earth, encompassing a roughly cylindrical grid in geospace. Details about the LFM grid can be found in the work of Lyon et al. [2004]. The LFM takes observed solar wind values as the upstream boundary condition and requires supersonic flow at the side and downstream boundaries. The magnetospheric simulation couples to a two-dimensional, height-integrated ionospheric simulation at the $2.1 R_{E}$ inner boundary, details of which can be found in the work of Fedder et al. [1995]. A comprehensive description of the numerics and assumptions underlying the magnetospheric model is given in the work of Lyon et al. [2004].

[14] We applied the same plasma sheet criteria listed in Table 1 to the LFM magnetospheric volume to identify the MHD plasma sheet. A representative time step from the LFM simulation is shown in Figure 2. The figure shows the noon-midnight plane of the simulation with the Sun to the left. The logarithm of plasma density is displayed in a color map; the lowest densities (blue) are found in the lobes and the highest densities (red) are found in the magnetosheath. The gray surface shown antisunward of the location of the Earth (center of the black GSM coordinate axes) simply bounds the volume of points comprising the 3-D MHD plasma sheet defined by the criteria of Table 1 and does not correspond to any density values of the color bar. Shades of gray indicate bumps in the 3-D surface. We performed the majority of the simulation analysis, including generating Figure 2, with the CISM-DX software package [Wiltberger et al., 2005].
[15] We used 2 months of WIND observations, the interval from 23 February to 26 April 1996, as upstream boundary conditions for the LFM model. We chose this interval because it was statistically similar to the solar wind conditions when the plasma sheet observations, discussed in section 2, were made by Geotail. We discuss this similarity requirement in section 4 . Figure 3 shows the solar wind time series which was simulated. From top to bottom, the panels contain solar wind number density $\left(\# / \mathrm{cm}^{3}\right)$, speed $(\mathrm{km} / \mathrm{s})$, ram pressure $(n P)$, and the IMF $B_{Y, G S M}(\mathrm{nT}), B_{Z, G S M}(\mathrm{nT})$, and $B_{\text {total }}(\mathrm{nT})$. We split the 2-month duration into nine subintervals of approximately $6 \mathrm{~d}$ each for ease of running the simulations, and due to two WIND perigee passes during the latter half of the interval (the large gaps near 27 March and 18 April). To avoid numerical constraints on $B_{X}$ in the simulation coordinates $(S M)$ at the upstream boundary of the model, we set that component to zero for the duration of the simulation. $B_{X}$ generally plays a minor role in solar wind-magnetosphere coupling, as the bow shock does not amplify its magnitude at the subsolar point [Lyon et al., 2004].

[16] We identified the volume of the MHD simulation which fulfills our plasma sheet criteria for every available model time step, chosen to be every $\sim 1.5 \mathrm{~min}$. Judging by an inspection of the model plasma sheet velocities [see Guild et al., 2008], our $\sim 1.5$ min output cadence is short enough to sufficiently sample the fastest dynamical changes in the simulated plasma sheet. The MHD plasma sheet changes size and shape based on changes in the input solar wind parameters, most notably with the southward component of the IMF. During the two month simulated interval, 


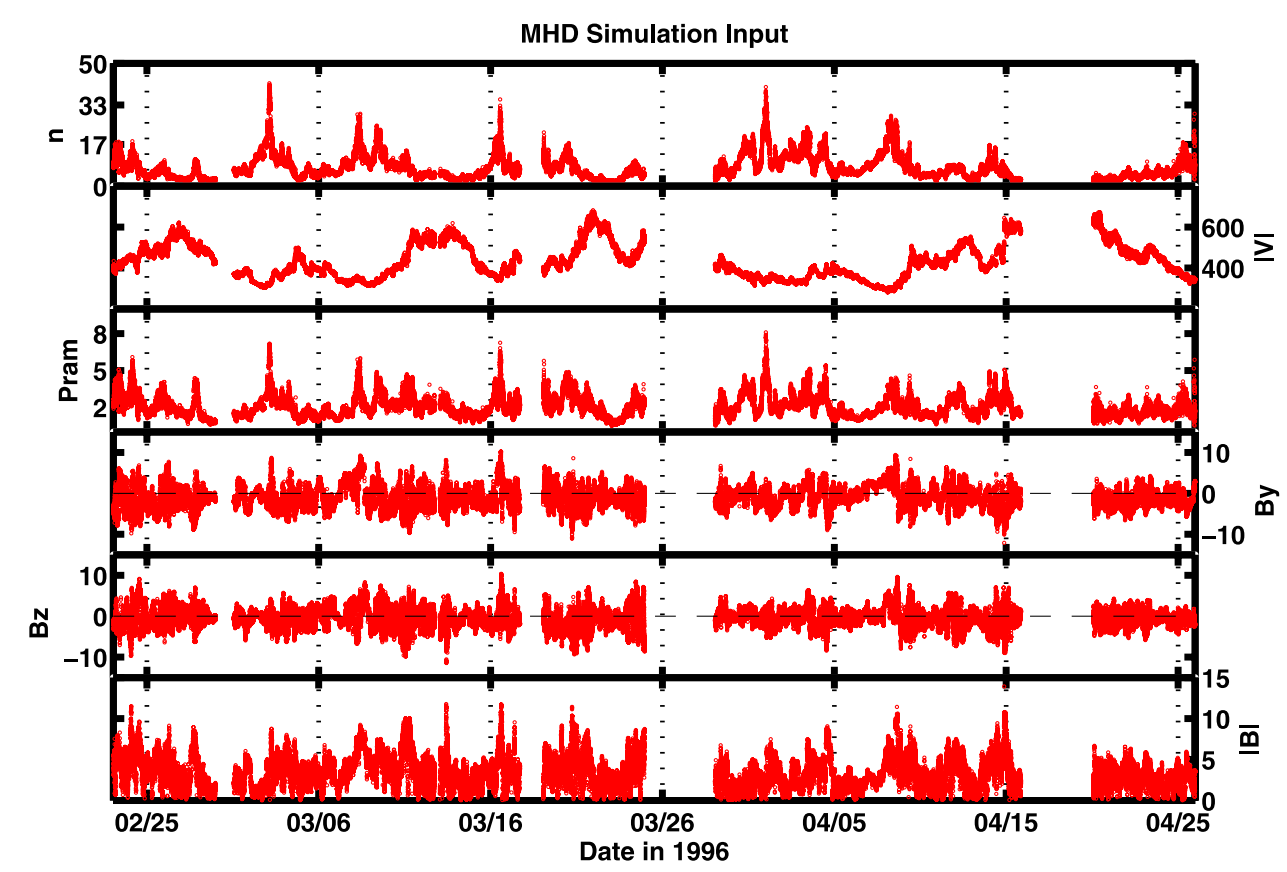

Figure 3. Two month solar wind time series driving the LFM model for this study. Shown are the solar wind density, speed, ram pressure, the $Y_{G S M}$ and $Z_{G S M}$ components, and the magnitude of the total magnetic field. The two large gaps in the time series near 27 March and 18 April correspond to WIND perigee passes and were not simulated.

the median antisunward extent of the plasma sheet was $\sim 28 R_{E}$, the median width in $Y_{G S M}$ was $28 R_{E}$, and the median thickness $\left(\Delta Z_{G S M}\right)$ was $13 R_{E}$. We averaged $\sim 10^{8}$ simulation points during the two month simulation to produce equatorial maps of MHD parameters to compare with corresponding maps from the Geotail observations within the plasma sheet.

\section{Data/Model Comparison Applicability}

[17] A direct comparison of the observed and simulated plasma sheet of this study would require a $\sim 6$-year global MHD simulation. Computational feasibility currently precludes simulating this long of an interval. Rather, in this study, we compared the statistical plasma sheet parameters observed by Geotail and simulated by the LFM. To ensure a reliable comparison of plasma sheet variables from both studies, we must first ensure statistical similarity of the solar wind drivers in each data set.

[18] We show distributions of upstream solar wind parameters driving both the real magnetosphere (red) and the simulated magnetosphere (blue) in Figure 4. The MHD input distributions are histograms of the solar wind for the entire 2-month simulated interval (blue lines). We used the solar wind observations merged with our plasma sheet data set (described in section 2) to plot the red distributions. The solar wind density (Figure 4a), velocity (Figure 4b), ram pressure (Figure 4c), and IMF $B_{Y}$ (Figure $4 \mathrm{~d}$ ), and $B_{Z}$ (Figure $4 \mathrm{e}$ ), are parameters key to controlling the magnetosphere (i.e., "geoeffective"). We also show the $D_{S T}$ index measured during the observed and simulated intervals in Figure $4 \mathrm{f}$ to demonstrate that both studies experienced a comparable level of geomagnetic storm activity. Note the similarity of geoeffective solar wind drivers between the observations (red curves of Figure 4) and simulation (blue curves of Figure 4). This suggests that the virtual magnetosphere is neither vastly overdriven nor underdriven relative to the real magnetosphere.

[19] To better quantify comparative magnetospheric forcing, we turned to a solar wind/magnetosphere coupling function, which can provide some measure of the solar wind's ability to energize the magnetosphere. One of the first examples of such a coupling function is the $\epsilon$ parameter, introduced by Perreault and Akasofu [1978]. The $\epsilon$ parameter has been interpreted as the upstream Poynting flux incident on the subsolar magnetopause and serves as a proxy for the solar wind energy input to the magnetosphere.

[20] We used the $\epsilon$ parameter to estimate the power input to our Geotail observational and LFM simulation databases. We took the original [Perreault and Akasofu, 1978] formulation:

$$
\epsilon=V B^{2} l_{0}^{2} \sin ^{2}\left(\frac{\theta}{2}\right)
$$

defining $V$ as the solar wind speed, $B$ as the IMF magnitude, $l_{0}^{2}$ as the effective cross-sectional area of the magnetosphere $\left(49 R_{E}^{2}\right.$ ), and $\theta$ as the clock angle of the IMF components in a plane perpendicular to the Sun-Earth line $\left(\theta=\operatorname{atan}^{-1}\left(\frac{B_{Y}}{B_{Z}}\right)\right)$. Judging by the similarity of the solar wind distributions shown in Figure 4, one might naively expect the $\epsilon$ distributions to match closely. We plot distributions of the $\epsilon$ parameter for both the Geotail (red) and LFM (dotted blue) studies in Figure 5. Both distributions are normalized to the area under their respective curves on a linear $\epsilon$ scale 

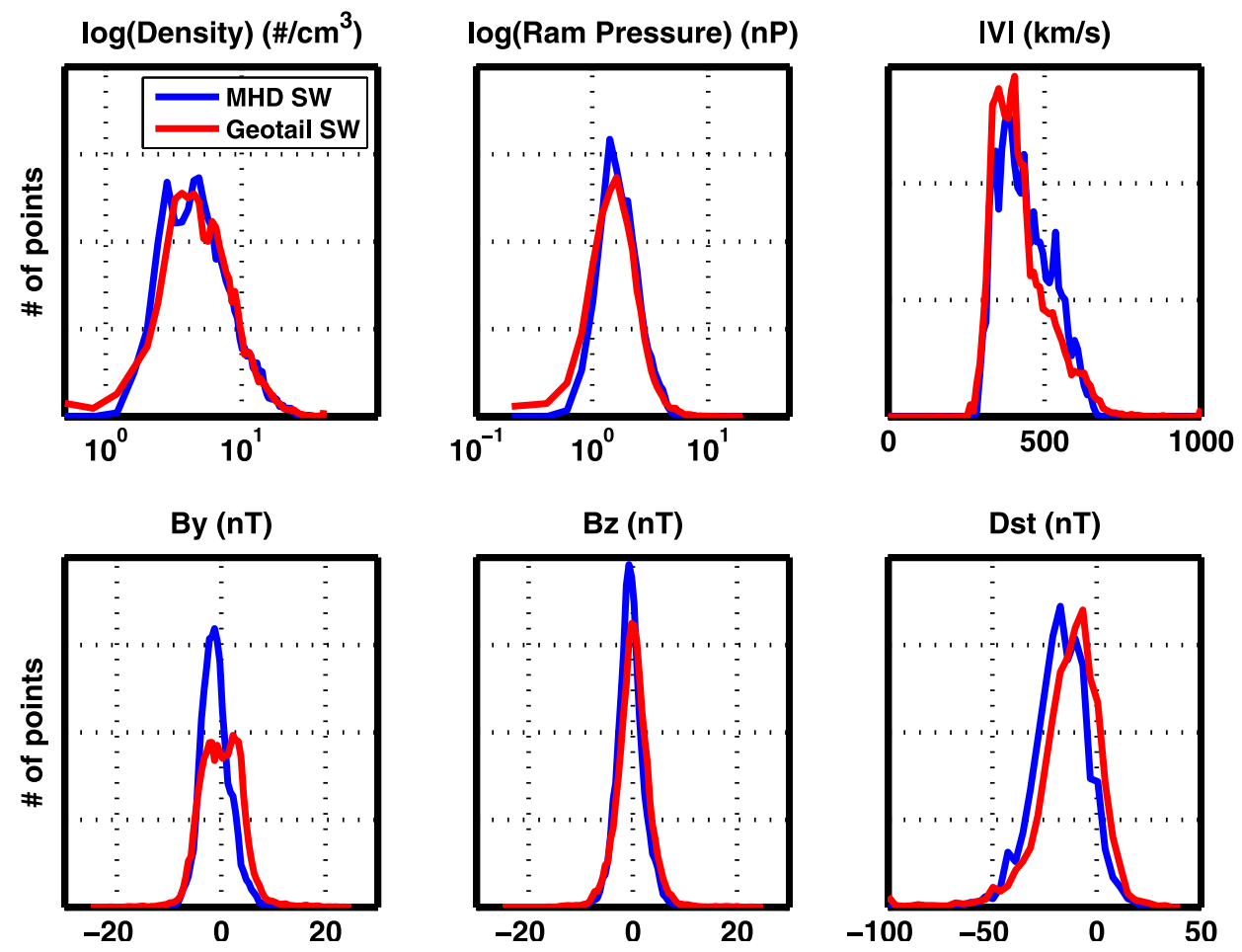

Figure 4. Distributions of solar wind parameters observed during the Geotail plasma sheet passes (red lines) and input to the LFM simulation (blue lines). Shown are histograms of $\log ($ Density $), \log \left(P_{R A M}\right), V$, $B y, B z$, and $D_{S T}$. Each set of distributions are normalized to the area under the curves; the peak Geotail (LFM) histograms typically have $\sim 10^{5}\left(\sim 10^{3}\right)$ samples.

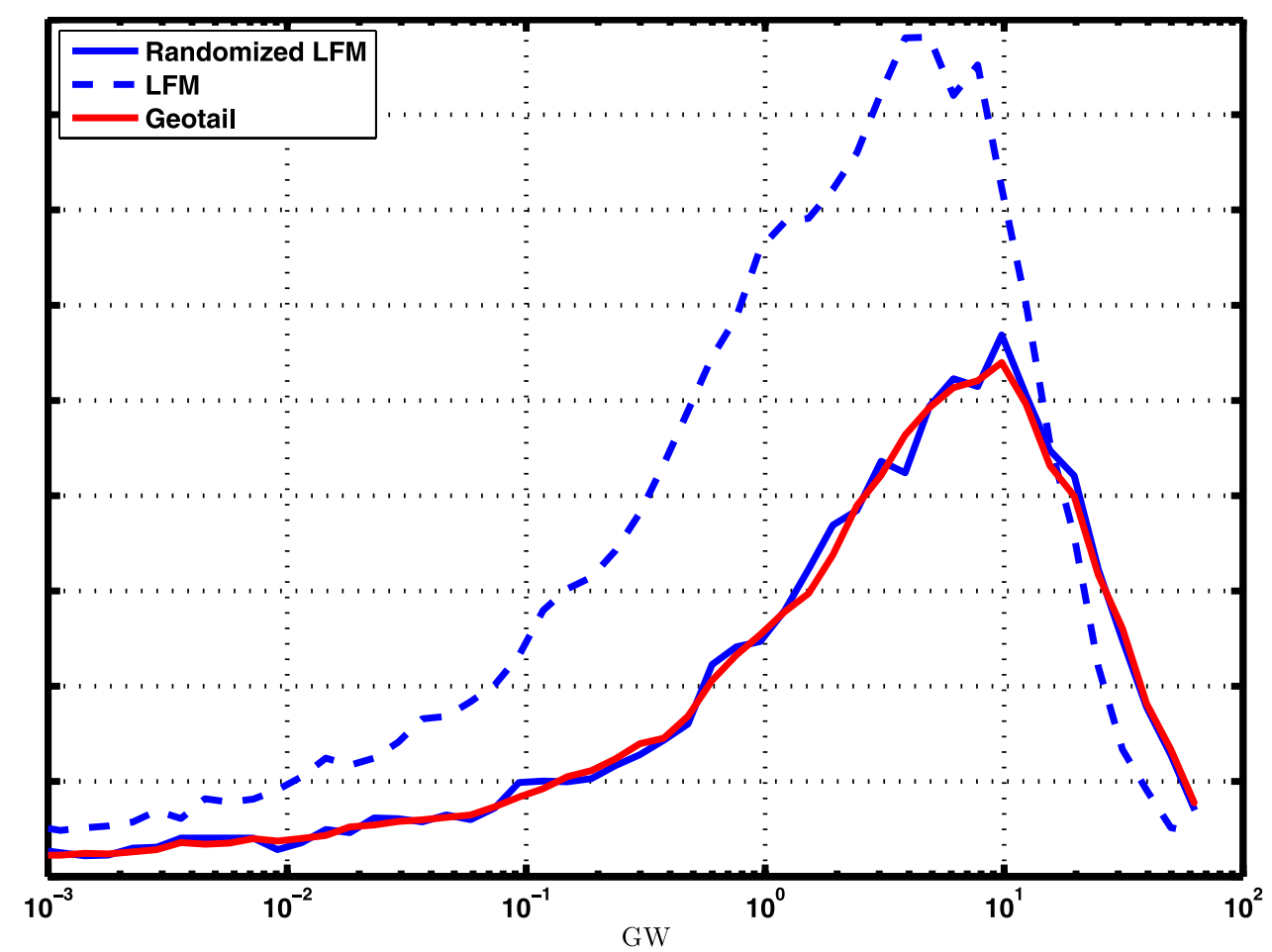

Figure 5. Comparison of $\epsilon$ values for the observations and simulations. Two months of solar wind inputs to the LFM are shown as a dashed blue curve (mean $\epsilon=4.6 \mathrm{GW}$ ) and 12 randomly selected days are plotted as the solid blue curve, which were selected to match the solar wind input to the observational database, plotted in red (mean $\epsilon=7.8 \mathrm{GW}$ ). 

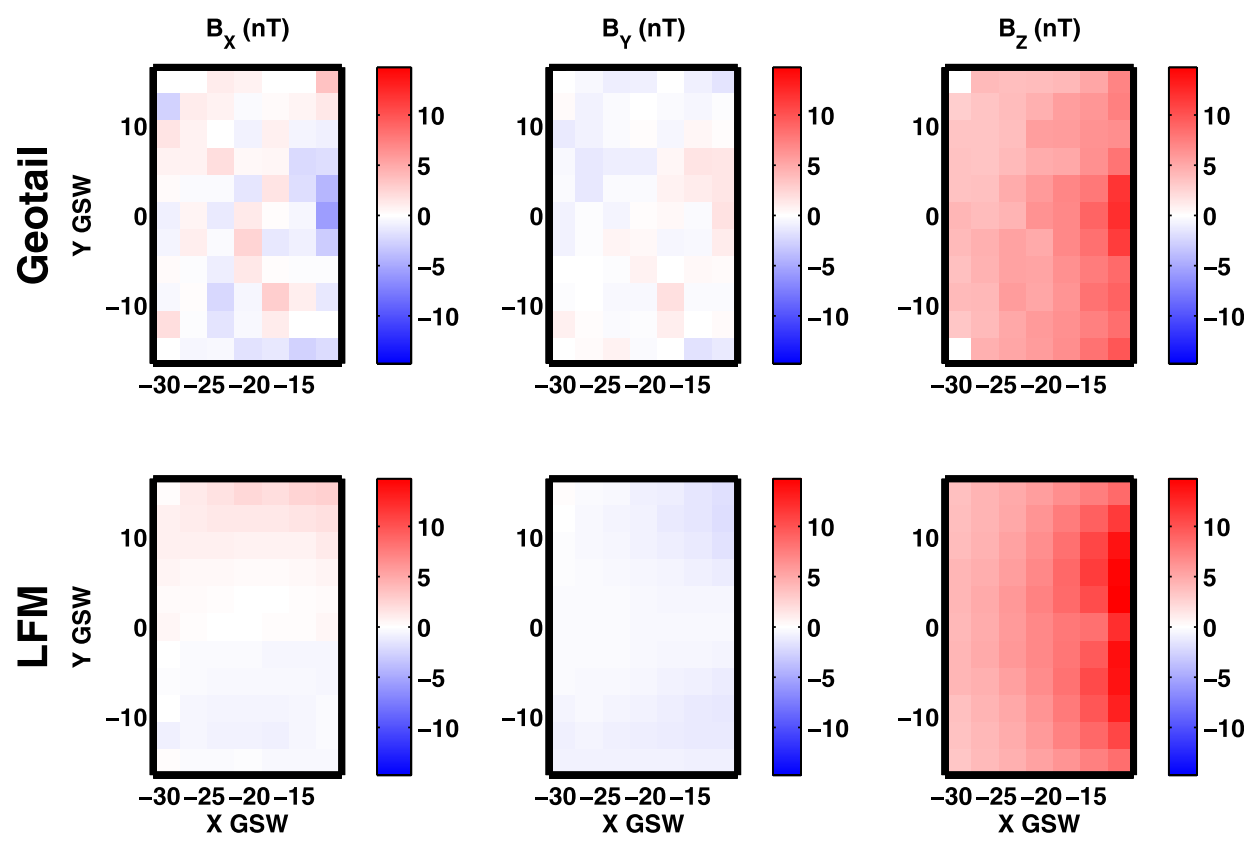

Figure 6. Equatorial maps of the mean magnetic field components (top) observed by Geotail and (bottom) simulated by the LFM. All panels have identical color scales. In both studies the largest field component is $B_{Z}$, as expected in a central plasma sheet database.

but are plotted here with the x-axis scaled logarithmically and in units of $10^{9} \mathrm{~W}$, or $\mathrm{GW}$. Important differences are obscured between the observations (red line) and the simulation (dotted blue line) due to logarithmically spaced bins in $\epsilon$, where small differences in the largest bins are compensated by large differences in the smallest bins to conserve the area under the distributions. Regardless, mismatched distributions indicate that the LFM is subject to less energetic (in terms of $\epsilon$ ) solar wind than the Geotail data set. The mean $\epsilon$ of the simulation is $4.6 \mathrm{GW}$, whereas the mean $\epsilon$ in the observational study is $7.8 \mathrm{GW}, 70 \%$ larger.

[21] To redress this $\epsilon$ mismatch, we first omitted all observed times with $\epsilon>100 \mathrm{GW}$, as the 2-month simulation contains no corresponding highly geoeffective solar wind. More importantly, we then randomly selected time steps during the 2-month simulation in proportion to the desired $\epsilon$ distribution calculated from the observational database (the red line in Figure 5). Taking care to use the maximum possible simulation duration without duplicating time steps, we arrived at the resampled solid blue distribution in Figure 5. The resampled simulation database comprised only $\sim 12$ total days of simulation time but much more closely reproduced the distribution of $\epsilon$ observed for the Geotail data set. Randomizing and selecting certain LFM time steps does not appreciably change the single-parameter solar wind distributions shown in Figure 4 (randomization not shown), another assessment of the statistical robustness of our comparison study. Given these similarly shaped distributions of geoeffective parameters such as $|V|, P_{\text {ram }}$, $B_{Z}$ and $\epsilon$, we can more reliably compare the plasma sheet samples from both studies in a statistical way. We perform this comparison for a variety of plasma and field quantities in the following section.

\section{Data/Model Comparison}

\subsection{Magnetic Fields}

[22] The magnetic topology within the magnetosphere orders many of the plasma populations near the Earth. Consequently, obtaining a consistent plasma sheet magnetic signature is an important first step in our comparison between the model and observations. We show in Figure 6 equatorial maps of Geotail (LFM) magnetic field components on the top (bottom) row. The equatorial grid contains $3 \times 3 R_{E}$ bins and extends from $-10 R_{E}<X_{G S M}<-30 R_{E}$ and $-15 R_{E}<Y_{G S M}<+15 R_{E}$. The columns display magnetic field components in $G S W$ coordinates, with color scales that are identical and symmetric about $0 \mathrm{nT}$.

[23] Figure 6 provides an important independent check of our plasma sheet identification criteria. We show that the mean value of the $Z$ component of the field (Figures $6 \mathrm{c}$ and 6f) is everywhere positive, as expected from a central plasma sheet sampling. In fact, the ratio of the equatorial $\left(B_{X Y}=\sqrt{B_{X}^{2}+B_{Y}^{2}}\right)$ to northward $\left(B_{Z}\right)$ field component can be interpreted as a model-independent measure of the proximity of the Geotail data set to the average location of the neutral sheet, with small (large) values close to (far from) the neutral sheet. The average field ratio in the equatorial plane is $0.82 \pm 0.16$, indicating the $Z_{G S W}$ dominates the equatorial components, and confirms that our selection criteria indeed excluded most observations made at higher latitudes within the plasma sheet, leaving only those on predominantly $Z_{G S W}$ field lines close to the neutral sheet. Moreover, the equatorial components of the magnetic 

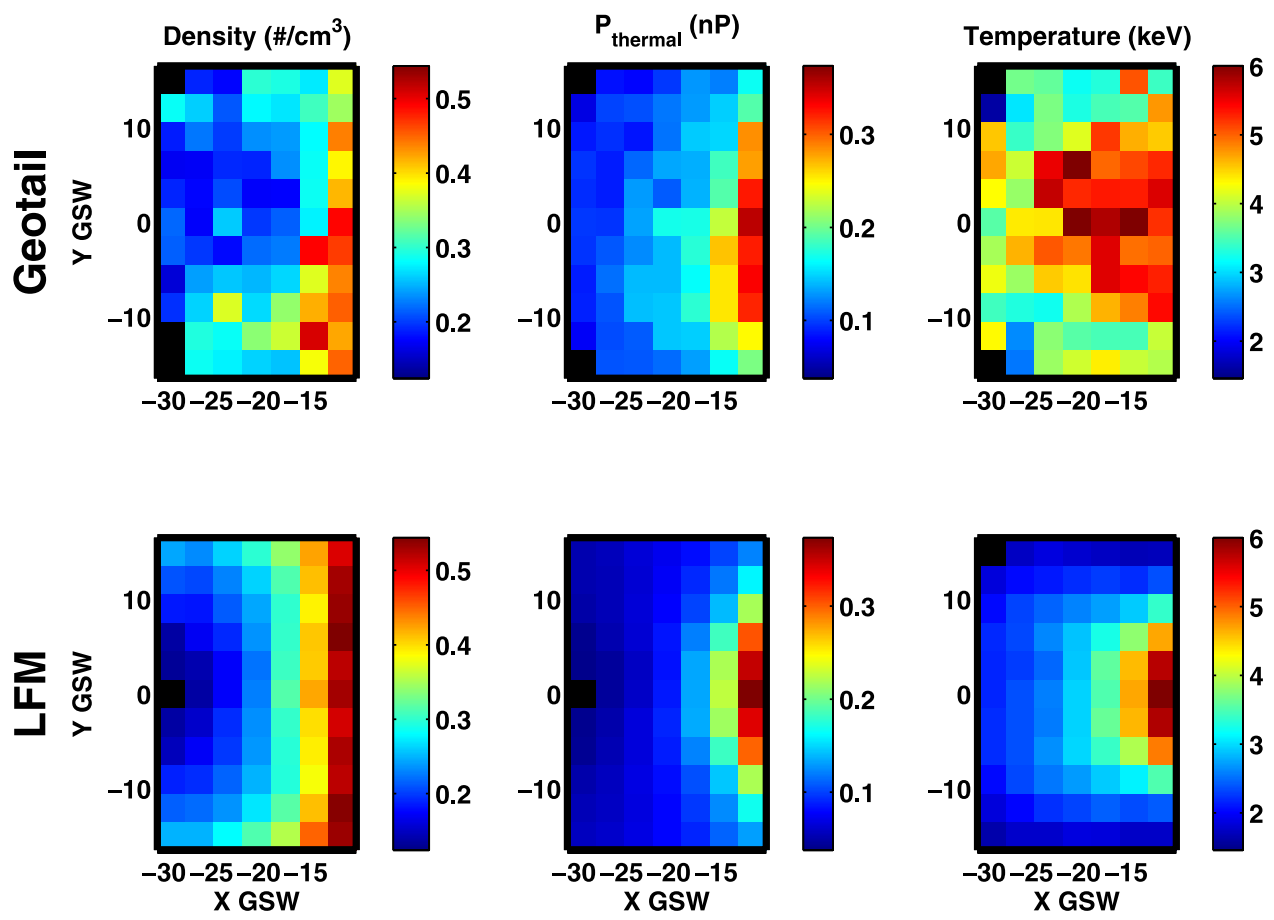

Figure 7. Equatorial maps of the mean plasma parameters (top) observed by Geotail and (bottom) simulated by the LFM. Shown are the density $\left(\mathrm{cm}^{-3}\right)$, thermal pressure $(\mathrm{nP})$, and thermal energy $(\mathrm{keV})$. The color scales for each pair of comparison maps is identical.

field in Figures $6 \mathrm{a}$ and $6 \mathrm{~b}$ exhibit mean values that are less than the corresponding $B_{Z}$ value.

[24] The Geotail $B_{Z}$ map (Figure 6c) is asymmetric in the dawn-dusk direction, with slightly larger $B_{Z}$ values in the dawn sector. This field asymmetry has been observed with various spacecraft [e.g., Fairfield, 1986; Wang et al., 2006] and can provide dawn-dusk pressure balance against a duskside thermal pressure enhancement caused by ions preferentially drifting duskward, as proposed by Spence and Kivelson [1993]. This asymmetry is not likely due to an orbital bias, as we found a similar $B_{Z}$ asymmetry with a much longer Geotail data set (11-year duration) with improved spatial coverage.

[25] Having ensured our magnetic field observations are indeed representative of the central plasma sheet, we can reliably compare them to the LFM field configuration in the bottom row of Figure 6 . The $Z$ component of the LFM field (Figure 6f) is dawn-dusk symmetric, in contrast to the slightly asymmetric Geotail map. The equatorial components of the LFM field are smaller than the corresponding field components in the Geotail study, and make up a smaller percentage of the total field. The mean ratio of $B_{X Y}$ to $B_{Z}$ is $0.72 \pm 0.10,13 \%$ smaller than this ratio derived from Geotail data. Even so, both the Geotail and MHD field component ratios lie within each others standard deviation error bars. This implies that we sampled both the Geotail and LFM plasma sheets at similarly small distances from their respective neutral sheets. Given this similar sampling, we undertake a comparison of plasma parameters in the next section.

\subsection{Plasma Parameters}

[26] We demonstrated in section 5.1 that both our Geotail and LFM studies yield the expected magnetic field signatures of the central plasma sheet. Having validated our method, we compare equatorial distributions of the plasma parameters in both studies in this section. Figure 7 displays the the mean number density $\left(\# / \mathrm{cm}^{3}\right)$, thermal pressure $(\mathrm{nPa})$, and thermal energy $(\mathrm{keV})$ equatorial maps in each column from left to right, using the same grid as in Figure 6. The color scales for each column are identical. We find mean Geotail parameters in the plasma sheet (top row of Figure 7) which are comparable to previously published work by other authors. Specifically, the midnight meridian pressure values are similar to those reported by Spence et al. [1989] in the region earthward of $X<-15 R_{E}$, but slightly underestimate their values tailward of that location. These pressures are smaller than those reported by Wang et al. [2004], likely because of the differences in plasma sheet criteria between these studies. Our observed density, pressure, and thermal energy maps also show similar distributions to the recent [Tsyganenko and Mukai, 2003] empirical plasma sheet model. The midlevel density $\left(0.35 \mathrm{~cm}^{-3}\right.$, green) of Figure 7a shows a trend that may indicate larger values near the flanks, consistent with equatorial density distributions in their Figure 10 (although the flank densities in our study are asymmetric, with a denser dawn flank, consistent with Wang et al. [2006]). Both pressure and thermal energy maps show a peak near the midnight meridian, again consistent with the distributions and values predicted by Tsyganenko and Mukai [2003] for the average solar wind parameters merged with our Geotail data set. 
[27] The columns of Figure 7 compare the Geotail and LFM mean plasma sheet parameters. The LFM density (Figure 7d) is larger than the corresponding Geotail density (Figure 7a) at the earthward boundary of the equatorial grid $\left(X_{G S W}>-12 R_{E}\right)$, for all $Y_{G S W}$. Even with the earthward density overestimate, it falls off more quickly with decreasing $X_{G S W}$, and by integrating the density over the equatorial grid in each study, we find that the LFM plasma sheet only contains $10 \%$ more mass than the corresponding Geotail plasma sheet. Midlevel density values $\left(0.35 \mathrm{~cm}^{-3}\right.$, green in Figure 7 d) show a noticeable trend of larger densities near the dawn and dusk flanks, a trend reminiscent of the Tsyganenko and Mukai [2003] density maps.

[28] The observed and simulated thermal pressure maps (Figures $7 \mathrm{~b}$ and $7 \mathrm{e}$ ) show similar peak magnitudes of 0.37 and $0.35 \mathrm{nP}$ for the LFM and Geotail, respectively. The maxima occur at the innermost edge of the noon-midnight meridian, at $X_{G S W}=-12 R_{E}$. The LFM pressure along the midnight meridian falls off slightly more steeply with $X_{G S W}$ distance, underestimating the Geotail pressure by a factor of $\sim 2.5$ at $X_{G S W}=-30 R_{E}$. The Geotail thermal pressure observations are fairly dawn-dusk symmetric, balanced by a dense dawn-side and hot dusk-side plasma sheet. These distributions do not show a significant dawn-dusk asymmetry as seen in the work of Wang et al. [2006], as such an effect would be overwhelmed by convection [e.g., Spence and Kivelson, 1990] during the significantly driven periods included in this study. The LFM thermal pressure map is symmetric about the noon-midnight meridian because the model lacks the physics to support significant preferentially westward drifting plasma.

[29] Comparisons of the thermal energy maps (Figures $7 \mathrm{c}$ and $7 \mathrm{f}$ ) show a dramatic difference in the equatorial area of hot plasma. The observations show a significantly larger region of $k T>4 \mathrm{keV}$ plasma, centered around the midnight meridian. The distribution is slightly asymmetric about the $X$ axis, recording hotter values in the dusk sector. Again, this asymmetry has been noted before and is typically attributed to energy dependent ion drifts enhancing the average energy of the dusk-side inner plasma sheet. The majority of the Geotail grid contains thermal energies $>3.5 \mathrm{keV}$. The LFM, however, has a small hot region near the inner boundary of the grid but the majority of the grid contains thermal energy $<3.5 \mathrm{keV}$. The LFM thermal energy shows no dawn-dusk asymmetry. Integrating the thermal energies of both plasma sheet studies indicate that the LFM generally underestimates plasma sheet temperatures by a factor of $\sim 3$.

[30] In summary, the comparisons made in Figure 7 show that the LFM is statistically denser and cooler than the average Geotail measurements in the central plasma sheet. The model does not capture the density and thermal energy dawn-dusk asymmetries, likely because energy dependent ion drifts are not included in the single-fluid ideal MHD equations. This combination of a density overestimate and thermal energy underestimate yields a favorable thermal pressure comparison between the observations and simulation. This thermal pressure is an important controlling parameter of large-scale plasma sheet dynamics, so our favorable comparison nevertheless gives us confidence in the global velocity comparisons in section 5.3. The LFM thermal pressure falls off slightly more steeply than the corresponding Geotail pressure, underestimating it by a factor of $\sim 2.5$ at the tailward edge of our equatorial grid.

\subsection{Velocity}

[31] On climatological timescales, the majority of impulsive plasma sheet dynamics average out to yield the classic magnetospheric convection flow pattern as first evinced by Dungey [1961]. To assess the LFM's ability to reproduce this proxy for global magnetic flux circulation, we compared the model mean perpendicular flows in the equatorial plane with the corresponding Geotail observations. Perpendicular flows (perpendicular to the instantaneous magnetic field) are indicative of moving flux tubes, and thus convective transport in the plasma sheet. Figure 8 shows mean $V_{\perp_{X Y}}$ vectors derived from Geotail data (Figure $8 \mathrm{a}$, red vectors) and from the LFM simulation (Figure 8b, blue vectors). A vector with a $5 R_{E}$ amplitude corresponds to a velocity of $100 \mathrm{~km} / \mathrm{s}$.

[32] Figure 8 exhibits an important discrepancy of our comparison between the model and the observations. The average magnitude of the flows in the Geotail (LFM) map is 38 (74) $\mathrm{km} / \mathrm{s}$, a factor of $\sim 2$ different. The average magnitude of the LFM flows are taken at the equatorial plane, then averaged over all 12 days of time steps making up Figure 8. We show in section 6 that the systematic overestimation of transpolar potential in the LFM ionosphere is consistent with the gross speed overestimate shown here.

[33] The flows on the duskside of the Geotail map are $30 \%$ larger than the dawnside, whereas the LFM duskside flow is only $4 \%$ larger than the dawnside in Figure 8 . This flow asymmetry is generally attributed to sampling a gradient-curvature drifting population in the near-Earth plasma sheet [e.g., Angelopoulos et al., 1993; Hori et al., 2000]. The ideal MHD equations solved by the LFM, however, do not adequately capture this energy-dependent drift effect, likely resulting in its much smaller dawn/dusk flow asymmetry. The alternative phenomenon most likely responsible for the smaller LFM flow asymmetry is an ionospheric Hall conductance asymmetry included in the LFM which maps into the magnetosphere, first demonstrated by Wolf $[1970]$.

\section{Discussion: Magnetospheric Driving}

[34] In the previous section we quantified many of the similarities and differences between the LFM parameters and Geotail observations in the central plasma sheet. Summarizing, we found that the equatorial LFM plasma sheet was slightly denser than observed and does not reproduce the observed dawn-dusk asymmetry. The simulated plasma sheet was cooler than observed, underestimating plasma sheet temperatures by $\sim 3$. The LFM thermal pressure, being the product of overestimated density and underestimated temperature had similar peak values at the earthward edge of the grid, but fell off more quickly with decreasing $X_{G S W}$ distance. The LFM convective flow patterns underestimated the observed duskward flow and overestimated the overall flow speed by a factor of $\sim 2$.

[35] We next investigate one possible cause for the large average flow magnitude discrepancy shown in Figure 8. Since we tailored the simulation interval to reproduce 

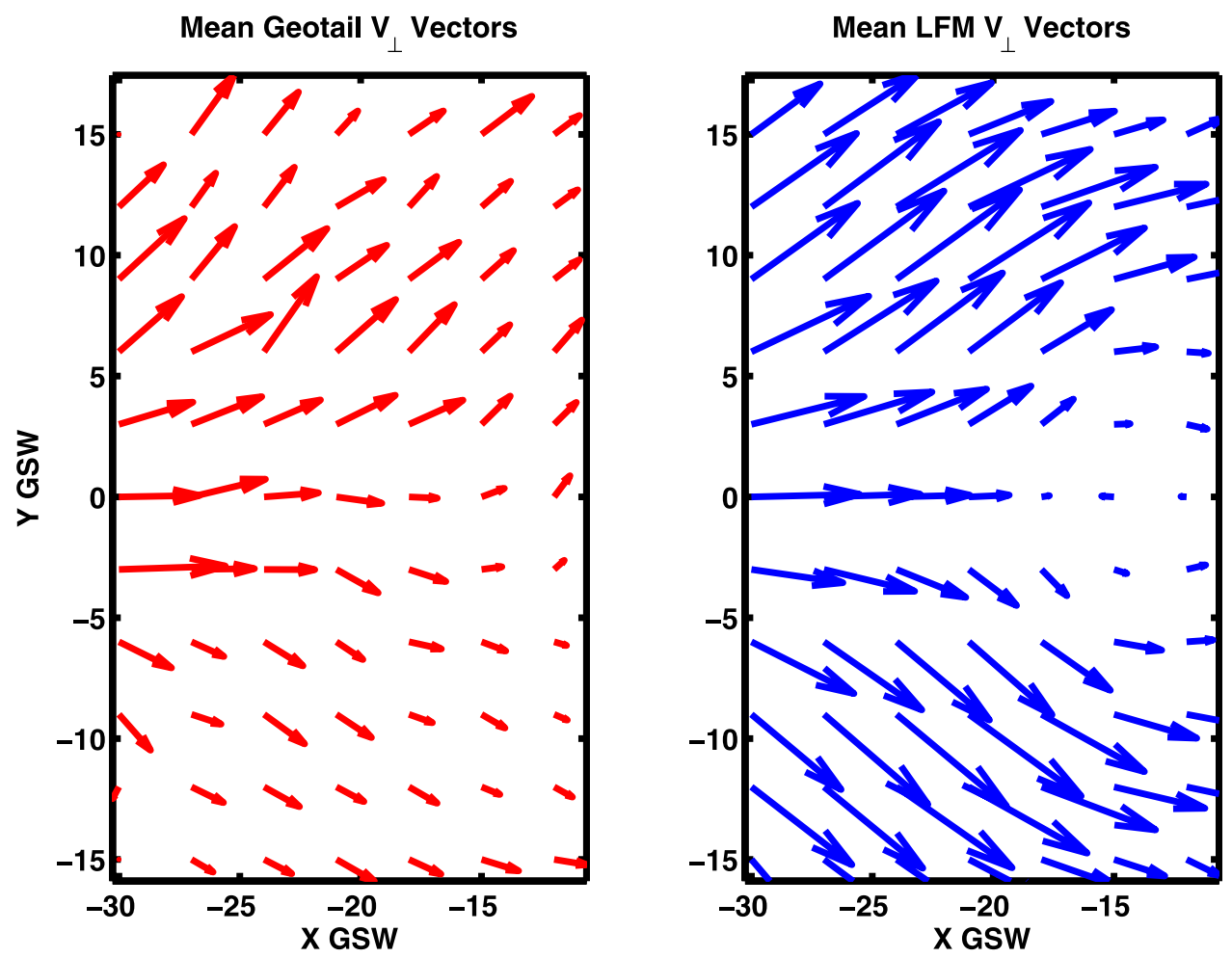

Figure 8. Comparison of the mean equatorial perpendicular flows (left) observed by Geotail and (right) simulated by the LFM. The average flow speed in the LFM is $2 \times$ the average flow speed observed by Geotail and does not feature the significant dawn-dusk asymmetry exhibited in the Geotail results.

exactly the observed distribution of incident solar wind power, we can rule out a mismatched $\epsilon$ as a cause of the excessive flow speed in the LFM plasma sheet. We will, however, investigate the efficiency with which energy enters the LFM magnetosphere, as measured by the transpolar potential in section 6.1, and assess the effect this energization has on plasma sheet flows in section 6.2.

\subsection{Ionospheric Potential}

[36] By limiting our simulation to only the distribution of observed solar wind $\varepsilon$ values, we ensured the incident power available to energize the LFM plasma sheet is statistically identical to that which powers the observed flows. A necessary intermediate step, however, requires that the incident energy penetrate the magnetosphere, prior to energizing the plasma within. We used the ionospheric transpolar potential as a proxy for the fraction of solar wind energy which penetrates the magnetopause.

[37] Measuring the electric potential in the ionosphere is challenging for this long interval, so we adopted the latest version of a well-used, thoroughly validated empirical model to approximate the ionospheric electric potential [Weimer, 2005]. The Weimer model uses electric field observations from many polar passes of the DE-2 spacecraft, sorted by solar wind and IMF parameters to produce an average polar cap electric potential pattern. Using the observed solar wind density, velocity, IMF $B y, B z$, and the dipole tilt angle for the simulated 2 months (23 February to 26 April 1996), the Weimer model returned the average potential pattern for those inputs. We took the difference between the maximum and minimum potential in the northern polar cap as the transpolar potential, $\Phi_{P C \text {,Weimer }}$. We regarded the empirical Weimer model as being representative of observations in the polar cap for these times, as recent published comparisons have demonstrated [e.g., Rothwell and Jasperse, 2006].

[38] We also computed the transpolar potential of the LFM model $\left(\Phi_{P C, L F M}\right)$ in the northern polar ionosphere from the difference between maximum and minimum electric potentials. We compare in Figure 9 a representative 5-d time series (24-29 February 1996) of the transpolar potentials taken from the Weimer and LFM models. The transpolar potential derived from the Weimer (LFM) model is shown in red (blue). For this interval of the two month simulation, we find that the LFM consistently overestimated the potential for values larger than $\sim 80 \mathrm{kV}$. The Weimer model rarely predicted potentials greater than this level during this interval, while the LFM routinely exceeded this value. Overestimating the transpolar potential is a known problem common to many global MHD models [e.g., Winglee et al., 1997] and in the LFM may be partially due to an incomplete representation of ionospheric microphysics [Merkin et al., 2005]. Another possible reason for the LFM overestimate of $\Phi_{P C}$ could be the resistivity inherent in the numerical solution of the MHD equations on a grid. Even with identical distributions of $\epsilon$ in the upstream solar wind, this numerical resistivity could serve to reconnect excessive magnetic flux at the LFM subsolar magnetopause, overenergizing the model magnetosphere and manifesting this excess in the overestimate of the LFM transpolar potential. We leave this aspect of numerical solar wind-magnetosphere coupling for a future study. 


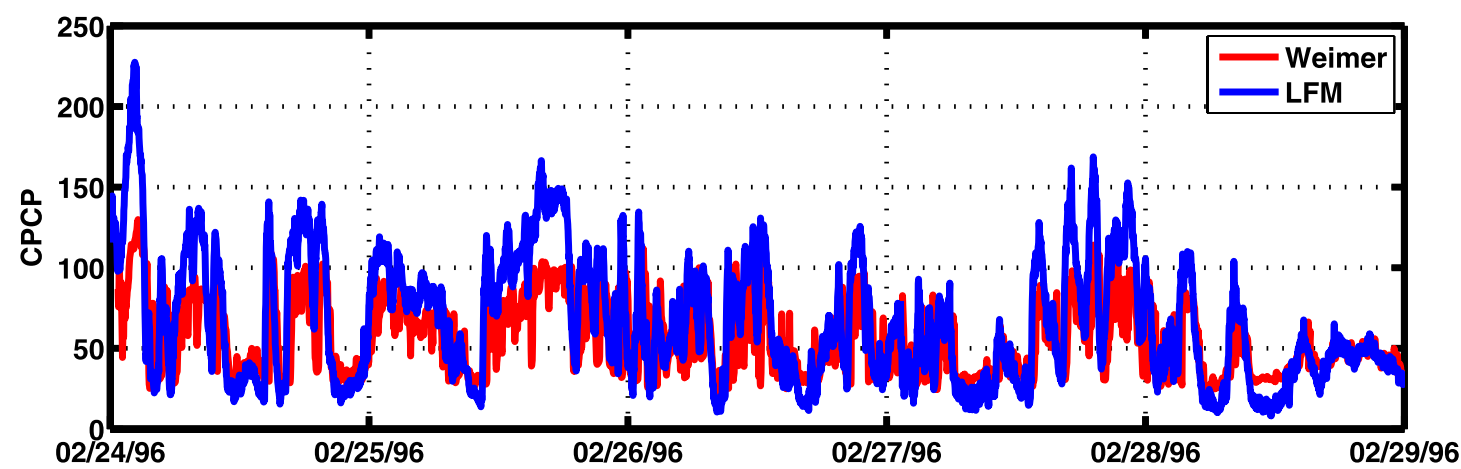

Figure 9. Comparison of $5 \mathrm{~d}$ of transpolar potentials taken from the Weimer (red) and the LFM (blue) models. Note the consistent LFM overestimate for $\Phi_{P C}>80 \mathrm{kV}$, especially from 24 to 26 February 1996. Also note the LFM underestimate for $\Phi_{P C}<25 \mathrm{kV}$, apparent in the first half of 28 February 1996.

[39] Alternatively, the $\Phi_{P C}$ discrepancy could be due to the Weimer model underestimating the true transpolar potential. Rothwell and Jasperse [2006] showed (in their Figure 6) that instead of the Weimer model underestimating the transpolar potential, it typically reproduced or exceeded DMSP observations of the transpolar potential during a magnetic storm. Therefore, given our 2-month interval chosen for low- to moderate-activity, the Weimer model is unlikely to systematically underestimate the transpolar potential (D. Weimer, private communication, 2006). The difference between the LFM and the Weimer models in our comparison is likely due to an overestimate on the part of the LFM.

[40] An illuminating way to compare the LFM and Weimer transpolar potentials over a long time period is with a scatterplot. We first smoothed the $\Phi_{P C \text {,Weimer }}$ and $\Phi_{P C, L F M} 2$-month time series with a 20 -min running average to filter out high frequency transients, then selected only the 12 randomized days used in the field, plasma, and flow comparisons of Figures 6, 7, and 8. We plot the resultant $\Phi_{P C, \text { Weimer }}$ versus $\Phi_{P C, L F M}$ in Figure 10 and color the points red (blue) for southward (northward) IMF $B_{Z}$. The dashed line in Figure 10 shows a unity slope, that is, if the LFM potential were identical to the Weimer potential. More than $2 / 3$ of all points fall below the dashed line, indicating that the LFM consistently computed a larger potential than the Weimer model for identical solar wind drivers. The linear equation which best fits the data is

$$
\Phi_{P C, \text { Weimer }}=0.48 \Phi_{P C, L F M}+24,
$$

and is plotted as a solid green curve. This linear fit indicates that on average, for $\Phi>50 \mathrm{kV}$, the LFM overestimates the true transpolar potential and that overestimation is larger for higher potentials. Conversely, for $\Phi<50 \mathrm{kV}$, the points lie above the dashed line and the LFM underestimates the true cross polar cap potential. The majority of low potentials corresponded to northward IMF, and as such, were not associated with effectively transferring solar wind energy to the magnetosphere, and ultimately the plasma sheet.

[41] This linear relationship between $\Phi_{P C, L F M}$ and $\Phi_{P C \text {, Weimer }}$ (equation (2)) has far more utility than just quantifying a trend. It allows us to rescale anomalous LFM potentials to a more reasonable range based on observations. In fact, by using a relationship between the ionospheric transpolar potential and the corresponding plasma sheet flow speed (shown next in section 6.2), equation (2) can also be used to rescale flow speeds. Normalizing ionospheric potentials could serve to reduce the disparity between simulated and observed flow speeds found in section 5.3. We examine how the plasma sheet speed varies with transpolar potential in the following section.

\subsection{Relationship of $\Phi_{P C}$ to Plasma Sheet Velocity}

[42] Having demonstrated that comparatively more energy is penetrating the LFM magnetosphere relative to the Weimer model (observations), we here show this excess energy serves in part to drive plasma sheet flows. We obtained a step-by-step correspondence of $\Phi_{L F M}$ and plasma sheet speed by first finding the average perpendicular velocity maps during the 2 months of LFM simulation. We averaged all flows on the equatorial grid to obtain one average flow magnitude per timestep. After smoothing the velocities with a 20-min running average and randomly selecting only those $12 \mathrm{~d}$ used to generate Figure 8, we show in Figure 11 the average LFM plasma sheet speed as a function of the transpolar potential. A clear trend exists indicating that faster plasma sheet flows correspond to larger transpolar potentials. We found that the data are best fit (lowest RMS error) with a quadratic equation,

$$
|V|=-0.0028 \Phi_{P C, L F M}^{2}+1.3 \Phi_{P C, L F M}-8.4 \text {. }
$$

given in the top left of the figure. The significant scatter in Figure 11 does not yield significant RMS error improvements with higher-order fits, so we adopt equation (3) for the remainder of this section.

[43] Taking advantage of the interrelationships between $\Phi_{P C, L F M}, \Phi_{P C, \text { Weimer }}$, and $|V|_{L F M}$ quantified in equations (2) and (3), we rescaled $\Phi_{P C, L F M}$ to the range of observed $\Phi_{P C, \text { Weimer }}$ potentials and calculated the expected plasma sheet velocities commensurate with the reduced LFM potential. We first normalized the $\Phi_{P C, L F M}$ potentials to the range of observed potentials using equation (2). During our 12-d random sample, the mean of the original $\Phi_{P C, L F M}=77 \mathrm{kV}$. For that same interval, $\left\langle\Phi_{\text {Weimer }}\right\rangle=62 \mathrm{kV}$. Rescaling the original LFM potential using equation (2) reduces the mean potential by $22 \%$ also, to $\left\langle\Phi_{L F M \text {,rescaled }}\right\rangle=62 \mathrm{kV}$. 


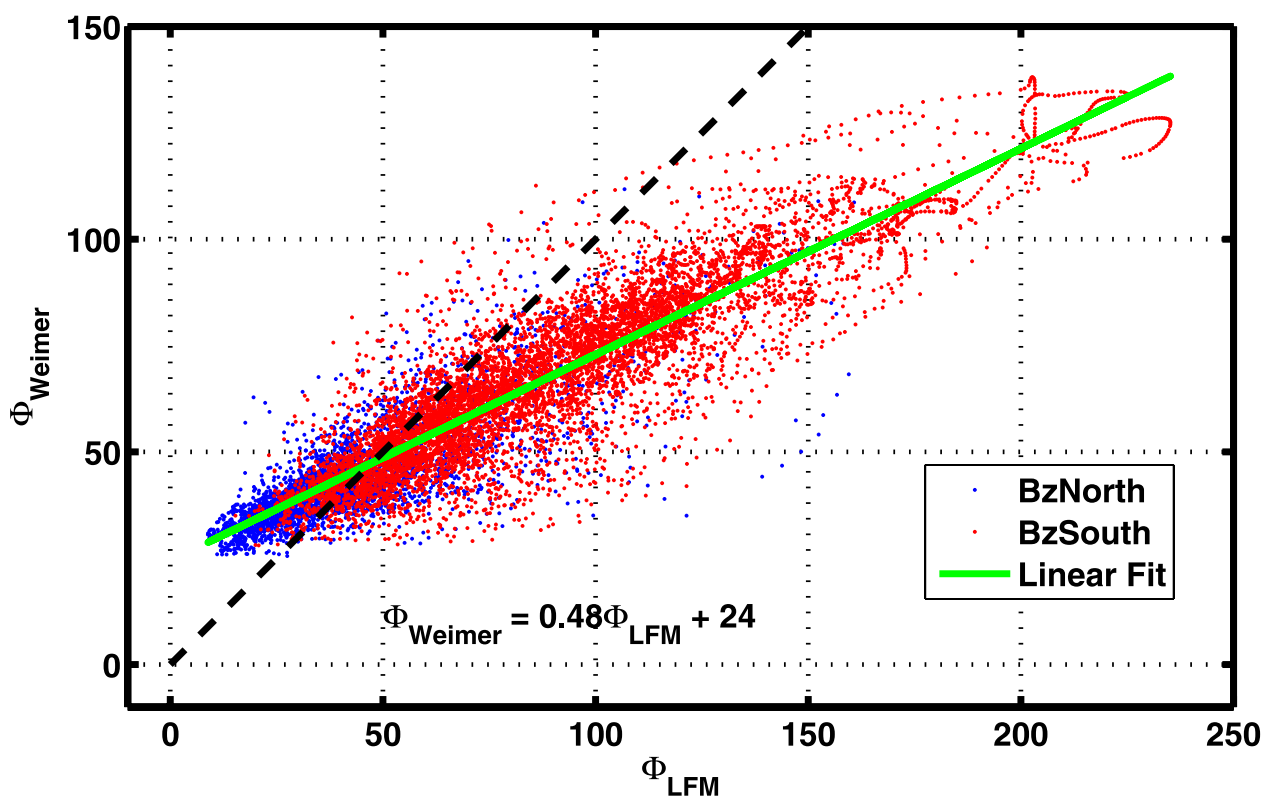

Figure 10. Scatterplot of the LFM $\Phi_{P C}$ (abscissa) plotted against the Weimer $\Phi_{P C}$ (ordinate). All potentials have units of $\mathrm{kV}$, and the dashed line highlights unity slope. Note the large number of points which fall below the dashed line, indicating an LFM underestimate of $\Phi_{P C}$. Red (blue) points correspond to southward (northward) IMF, and the linear fit is overplotted in green.

[44] We used equation (3) to calculate the expected flow speed given our rescaled LFM potential. Again, the average plasma sheet flow speed of twelve randomized days of LFM simulation is $74 \mathrm{~km} / \mathrm{s}$, a factor of $\sim 2$ larger than the corresponding Geotail observations. Substituting our nor- malized $\Phi_{P C, L F M}$ into equation (3), we found an average $|V|_{\text {rescaled }}$ of $64 \mathrm{~km} / \mathrm{s}$, a reduction of $\sim 15 \%$. Therefore correcting the LFM transpolar potential overestimate can only partially account for the elevated LFM plasma sheet flow speeds. Since the process of plasma sheet energization

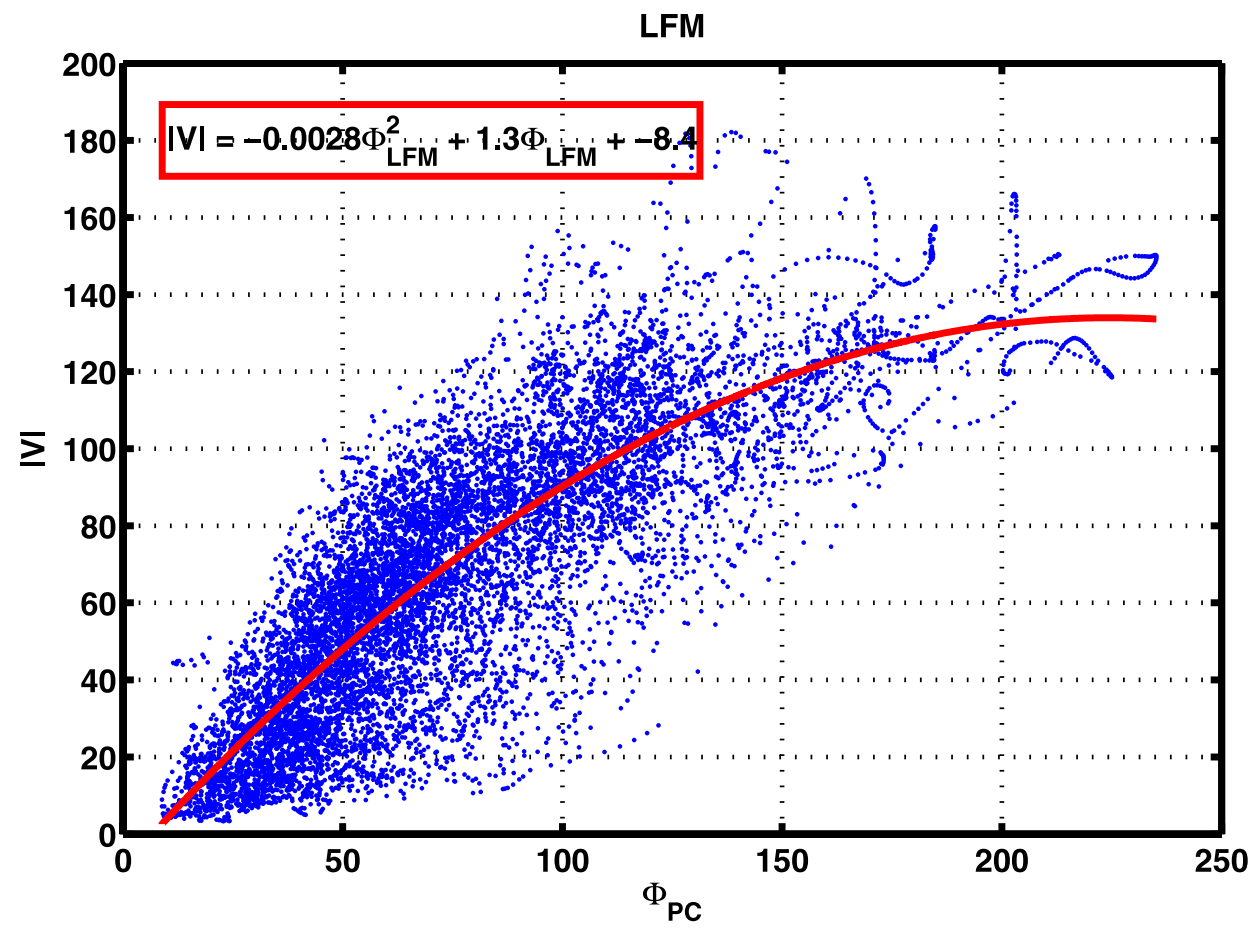

Figure 11. Scatterplot of the average LFM plasma sheet velocity (abscissa) plotted against the LFM $\Phi_{P C}$ (ordinate). A clear trend shows the average flow speed increases with increasing $\Phi_{P C}$. We fit the data with a quadratic polynomial (red) and give the best fit equation at top left. 
is a complex one, involving many transport paths and storage mechanisms, this simple analysis may be omitting an important contribution to the flow speed. For example, the average plasma sheet flow speed may also be controlled by the inherently transient nature of fast flows, which are averaged out in a statistical treatment. We pursue this line of investigation in a companion paper [Guild et al., 2008].

\section{Summary and Conclusions}

[45] This study represents a statistical, comprehensive validation of the LFM model in the plasma sheet. By comparing average properties of 6 years of Geotail observations with a 2 -month LFM simulation, we carried out a rigorous test of the model performance under a variety of solar wind conditions and quantified and investigated the reason for any discrepancies between the model and observations. For fair comparison, we ensured the statistical similarity of the solar wind inputs to both the real and virtual magnetosphere. We identified the central plasma sheet in the data and simulation by including only points which satisfy common criteria listed in Table 1. After verifying the expected magnetic field signature in our central plasma sheet studies, we compared equatorial maps of density, thermal pressure, thermal energy, and velocity. Generally, we found that the LFM reproduces many of the climatological features of the Geotail data set. We also find systematic model/data discrepancies; most notably, the LFM consistently overestimated the plasma sheet density close to the Earth and underestimated the temperature by a factor of $\sim 3$. We found the LFM overestimated the global average flow speed by a factor of $\sim 2$, and showed that excessive transpolar potentials in the LFM ionosphere likely contribute at least $15 \%$ to this overestimate. An important aspect of this study is the unbiased validation of a global MHD model with a statistical data set, and the establishment of standard benchmarks to measure future versions of the model against.

[46] Acknowledgments. TBG would like to acknowledge useful discussions with J. Hughes, D. Weimer, J. Raeder, T. Nagai, and T. Mukai. Geotail magnetic field and plasma data were provided by $\mathrm{T}$. Nagai and T. Mukai, respectively, through DARTS at the Institute of Space and Astronautical Science, JAXA in Japan. We would also like to thank $\mathrm{K}$. Ogilvie and R. Lepping for the plasma and magnetic field data from the WIND satellite and D. McComas and N. Ness for the plasma and magnetic field data from the ACE mission. All solar wind data was obtained via the Coordinated Data Analysis Web site. We appreciate the use of D. Weimer's latest series of models, contributed to the CISM validation effort. The simulations were performed on Boston University and NCAR computational resources. This material is based upon work supported in part by the National Science Foundation under grant DGE-0221680 and GEM grant ATM-0602708 and in part by CISM, which is funded by the STC Program of the National Science Foundation under agreement ATM-0120950.

[47] Wolfgang Baumjohann thanks Zoltan Voros, Donald Fairfield, and another reviewer for their assistance in evaluating this paper.

\section{References}

Angelopoulos, V., et al. (1993), Characteristics of ion flow in the quiet state of the inner plasma sheet, Geophys. Res. Lett., 20, 1711-1714.

Angelopoulos, V., et al. (1994), Statistical characteristics of bursty bulk flow events, J. Geophys. Res., 99, 21,257-21,280.

Baumjohann, W., G. Paschmann, and H. Luhr (1990), Characteristics of high-speed ion flows in the plasma sheet, J. Geophys. Res., 95, $3801-$ 3809.

Dungey, J. W. (1961), Interplanetary magnetic field and the auroral zones, Phys. Rev. Lett., 6, 47-48, doi:10.1103/PhysRevLett.6.47.
Fairfield, D. H. (1986), The magnetic field of the equatorial magnetotail from 10 to 40 Earth radii, J. Geophys. Res., 91, 4238-4244.

Fedder, J., J. Lyon, S. Slinker, and C. Mobarry (1995), Topological structure of the magnetotail as a function of interplanetary magnetic field direction, J. Geophys. Res., 100, 3613-3621.

Gleisner, H., and H. Lundstedt (1997), Response of the auroral electrojets to the solar wind modeled with neural networks, J. Geophys. Res., 102, 14,269-14,278, doi:10.1029/96JA03068.

Guild, T., H. Spence, L. Kepko, M. Wiltberger, C. Goodrich, J. Lyon, and W. Jeffrey Hughes (2004), Plasma sheet climatology: Geotail observations and LFM model comparisons, J. Atmos. Terr. Phys., 66, 13511360, doi:10.1016/j.jastp.2004.03.021.

Guild, T., H. Spence, L. Kepko, V. Merkin, M. Wiltberger, J. Lyon, and C. Goodrich (2008), Geotail and LFM comparisons of plasma sheet climatology 2: Flow variability, J. Geophys. Res., 113, A04217, doi:10.1029/2007JA012613.

Hammond, C., M. Kivelson, and R. Walker (1994), Imaging the effect of dipole tilt on magnetotail boundaries, J. Geophys. Res., 99, 6079-6092.

Hori, T., K. Maezawa, Y. Saito, and T. Mukai (2000), Average profile of ion flow and convection electric field in the near-earth plasma sheet, Geophys. Res. Lett., 27, 1623-1626.

Kokubun, S., T. Yamamoto, M. Acuna, K. Hayashi, K. Shiokawa, and H. Kawano (1994), The Geotail magnetic field experiment, J. Geomagn. Geoelectr., 46, 7-21.

Lyon, J. G., J. A. Fedder, and C. M. Mobarry (2004), The Lyon-FedderMobarry (LFM) global MHD magnetospheric simulation code, J. Atmos. Terr. Phys., 66, 1333-1350, doi:10.1016/j.jastp.2004.03.020.

Merkin, V. G., G. Milikh, K. Papadopoulos, J. Lyon, Y. S. Dimant, A. S. Sharma, C. Goodrich, and M. Wiltberger (2005), Effect of anomalous electron heating on the transpolar potential in the LFM global MHD model, Geophys. Res. Lett., 32, L22101, doi:10.1029/2005GL023315.

Mukai, T., S. Machida, Y. Saito, M. Hirahara, T. Terasawa, N. Kaya, T. Obara, M. Ejiri, and A. Nishida (1994), The low energy particle (LEP) experiment onboard the Geotail satellite, J. Geomagn. Geoelectr., 46, 669-692.

Nagai, T., et al. (1998), Structure and dynamics of magnetic reconnection for substorm onsets with Geotail observations, J. Geophys. Res., 103, 4419-4440, doi:10.1029/97JA02190.

Nishida, A., T. Mukai, T. Yamamoto, Y. Saito, S. Kokubun, and K. Maezawa (1995), GEOTAIL observation of magnetospheric convection in the distant tail at $200 R_{E}$ in quiet times, J. Geophys. Res., 100, 23,663-23,676, doi:10.1029/95JA02519.

Perreault, P., and S.-I. Akasofu (1978), A study of geomagnetic storms, Geophys. J., 54, 547-573.

Rothwell, P. L., and J. R. Jasperse (2006), Modeling the connection of the global ionospheric electric fields to the solar wind, J. Geophys. Res., 111, A03211, doi:10.1029/2004JA010992.

Spence, H. E., and M. G. Kivelson (1990), The variation of the plasma sheet polytropic index along the midnight meridian in a finite width magnetotail, Geophys. Res. Lett., 17, 591-594.

Spence, H. E., and M. G. Kivelson (1993), Contributions of the low-latitude boundary layer to the finite width magnetotail convection model, J. Geophys. Res. 98, 15,487-15,496.

Spence, H. E., M. G. Kivelson, R. J. Walker, and D. J. McComas (1989), Magnetospheric plasma pressures in the midnight meridian - Observations from 2.5 to $35 \mathrm{R}(\mathrm{E}), J$. Geophys. Res., 94, 5264-5272.

Tsyganenko, N. A., and D. H. Fairfield (2004), Global shape of the magnetotail current sheet as derived from Geotail and Polar data, J. Geophys. Res., 109, A03218, doi:10.1029/2003JA010062.

Tsyganenko, N. A., and T. Mukai (2003), Tail plasma sheet models derived from Geotail particle data, J. Geophys. Res., 108(A3), 1136, doi:10.1029/ 2002JA009707.

Vassiliadis, D., A. J. Klimas, D. N. Baker, and D. A. Roberts (1995), A description of the solar wind-magnetosphere coupling based on nonlinear filters, J. Geophys. Res., 100, 3495-3512.

Wang, C.-P., L. R. Lyons, T. Nagai, and J. C. Samson (2004), Midnight radial profiles of the quiet and growth-phase plasma sheet: The Geotail observations, J. Geophys. Res., 109, A12201, doi:10.1029/ 2004JA010590.

Wang, C.-P., L. R. Lyons, J. M. Weygand, T. Nagai, and R. W. McEntire (2006), Equatorial distributions of the plasma sheet ions, their electric and magnetic drifts, and magnetic fields under different interplanetary magnetic field $B_{z}$ conditions, J. Geophys. Res., 111, A04215, doi:10.1029/ 2005JA011545.

Weimer, D. R. (2005), Improved ionospheric electrodynamic models and application to calculating Joule heating rates, J. Geophys. Res., 110, A05306, doi:10.1029/2004JA010884.

Wiltberger, M., R. S. Weigel, M. Gehmeyr, and T. Guild (2005), Analysis and visualization of space science model output and data with CISM-DX, J. Geophys. Res., 110, A09224, doi:10.1029/2004JA010956. 
Wing, S., and P. T. Newell (1998), Central plasma sheet ion properties as inferred from ionospheric observations, J. Geophys. Res., 103, 67856800, doi:10.1029/97JA02994.

Winglee, R. M., V. O. Papitashvili, and D. R. Weimer (1997), Comparison of the high-latitude ionospheric electrodynamics inferred from global simulations and semiempirical models for the January 1992 GEM campaign, J. Geophys. Res., 102, 26,961-26,978, doi:10.1029/97JA02461.
Wolf, R. A. (1970), Effects of ionospheric conductivity on convective flow of plasma in the magnetosphere., J. Geophys. Res., 75, 4677-4698.

C. C. Goodrich, E. L. Kepko, J. G. Lyon, V. Merkin, and H. E. Spence, Center for Space Physics, Boston University, Boston, MA 02215, USA.

T. B. Guild, Space Sciences Department/Chantilly, The Aerospace Corporation, 15049 Conference Center Drive, CH3/210, Chantilly, VA 20151-3824, USA. (timothy.guild@aero.org)

M. Wiltberger, High Altitude Observatory, National Center for Atmospheric Research, 3450 Mitchell Lane, Boulder, CO 80301, USA. 